\title{
Eedmental \\ Upper GI bleeding - Has mortality changed with advancements in therapy?
}

\section{Rajesh Gupta, D Nageshwar Reddy}

Asian Institute of Gastroenterology, Upper gastrointestinal bleeding (UGIB) is one of the most common GI medical emergencies, Hyderabad, India which all GI units deal with everyday. UGIB is broadly categorized into variceal and non

Correspondence:

Dr D Nageshwar Reddy

Email: aigindia@yahoo.co.in variceal bleeding. This distinction becomes crucial as the management strategies are different for variceal and nonvariceal bleeding. There has been tremendous advancement in pharmacological and endoscopic interventions in management of upper GI bleeding over the past decade. However, it still carries considerable morbidity, mortality and health economic burden. Peptic ulcer bleeding is the predominant cause of nonvariceal bleeding in the US and Europe. Recent data indicate a decline in the incidence of peptic ulcer disease and peptic ulcer bleeding in both the United States and Europe, which can be attributed to a decrease in $\mathrm{H}$. pylori infection. Numerous studies from US, Europe and some Asian countries have reported peptic ulcer bleeding mortality 5-12\% $\%^{1,2}$. Furthermore, these peptic ulcer bleeding linked deaths are not a direct result of bleeding ulcers. Instead, death occurs due to multi organ failure and other associated co morbidities. These studies have also shown that endoscopic interventions and pharmacological treatments have reduced rebleeding rates, rates of surgical interventions, days of hospitalization and number of blood units transfused. However, none of these therapeutic approaches have reduced the overall mortality associated with the bleed event. Hence, there is a need to relook at factors associated with mortality and revise management strategies accordingly.

Foremost, reasons for failure to reduce mortality rates despite major advances in endoscopic hemostatic modalities needs to be explored. A number of factors have been proposed as possible causes of persistently high mortality associated with peptic ulcer bleeding .Age and the presence of associated comorbidities are the most important factors for high mortality in a bleeding event. Sung et al reported an overall mortality of $6.2 \%$ in patients with peptic ulcer bleeding in a large cohort of 9,375 patients $^{3} .80 \%$ of these deaths were not related to ulcer bleed as opposed to $20 \%$ of mortality directly relatedto peptic ulcer bleed. Among those who died of non-bleeding related causes, multi-organ failure (24\%), pulmonary causes $(23.5 \%)$ and terminal malignancies were the most common causes, while bleeding related deaths occurred when immediate control of bleed could not be achieved or failure of endoscopic therapy within $48 \mathrm{hrs}$. The mean age of patients who died of bleeding related causes was higher than those who died of non-bleeding related causes. (75.4 years Vs 71.7 years, $\mathrm{p}=0.01$ ). The survivors were younger with less severe comorbid diseases than non survivors. (61years vs 72.5 years). This data clearly indicate that pharmacological or endoscopic intervention will have little impact on overall mortality. Hence, the efforts should be focused on identifying non GI factors associated with poor outcomes and reduce the risk of multi organ failure, especially in elderly population.

How relevant is the above-mentioned scenario in the Indian context? Can we extrapolate the Western experience in India? What are the most common causes of UGI bleeding in India? What is overall prevalence of peptic ulcer bleeding? Do these patients have access to a high 
standard of care treatment? What are the management protocols in different parts of the country? Do we have reliable mortality data? We do not have satisfactory answers to any of these questions at present. In a previous study from AIIMS, Anand et al reported variceal bleed (45\%) as the commonest cause of UGI bleeding ${ }^{4}$. Similarly, a few studies from western and southern India have reported variceal bleeding as the commonest cause of UGIB. On the other hand, a few studies from different centers report duodenal ulcer as the commonest cause of upper GI bleeding .So we have conflicting data on UGIB from India. If we analyze these studies, the data represent a highly selective population and have a referral bias.

In the current manuscript, Singh et al have described their experience of UGIB from coastal Odisha ${ }^{5}$. In this study, the commonest causes of UGIB are duodenal ulcer (57.5\%) followed by esophageal varices $(13.0 \%)$. Out of 608 bleeders, $47 \%$ patients required hospitalization and $35 \%$ patients needed blood transfusion. However, this study does not mention how many patients' required endoscopic interventions or surgery. What was the outcome of these interventions? There is no mention of bleed related mortality either. Despite these limitations, the current study appears to represent the real life scenario in a defined geographical area.

At present, we have contradictory data on the etiological spectrum of UGIB in India. This fact emphasizes the need to generate more data on the spectrum of UGIB in different regions of the country. Until and unless, we have comprehensive data on the profile of UGIB, we can neither plan management strategies nor recommend any practice guidelines to have a meaningful impact on mortality. We cannot extrapolate the Western experiences in the Indian scenario. Of course, there has been rapid advancement in endoscopic interventions in different parts of India in last decade. But these facilities are not adequate and not accessible to a large population in India. Till date, majority of patients with UGIB are managed in general hospitals all over country. In most of these hospitals, the facility of emergency endoscopy is almost non existent.

Therefore, we have a long way to go in management of UGIB in India. In this scenario, it is difficult to assess the impact of advancement in therapy on UGI bleeding in India.

\section{References}

1. Lans Angel. Upper GI Bleeding -Associated Mortality: challenges to improving a resistant outcome. Am J Gastroenterol. 2010;105:90-2

2. Jairath V, Barkun A N. Improving outcome from acute upper gastrointestinal bleeding. Gut. 2012;61:1246-9

3. Sung JJ, Tsoi, Ma T et al. Causes of mortality in patients with peptic ulcer bleeding: a prospective cohort study of 10,428 cases. Am J Gastroenterol. 2010;105:84-9.

4. Anand AC, Tandan BN, Nundy S .The causes, management and outcome of upper gastrointestinal haemorrhage in an Indian hospital. Br J Surg.1983;70:209-11

5. Singh SP, Panigrahi MK. Spectrum of Upper Gastrointestinal Hemorrhage in coastal Odisha. (this issue) 